\title{
A Hybrid Iterated Greedy Algorithm for Job Shop Scheduling Problem
}

Shikui Zhao ( $\square$ me_zhaosk@ujn.edu.cn )

University of Jinan https://orcid.org/0000-0002-4702-5673

\section{Lin Huang}

University of Jinan

Qing Han

University of Jinan

\section{Research Article}

Keywords: Job shop scheduling problem , Tabu search , Iterated greedy algorithm , Deconstruction and reconstruction, Maximum completion time

Posted Date: March 1st, 2022

DOI: https://doi.org/10.21203/rs.3.rs-1290801/v1

License: (c) (1) This work is licensed under a Creative Commons Attribution 4.0 International License. Read Full License 


\section{Title page}

\section{A Hybrid Iterated Greedy Algorithm for Job Shop Scheduling Problem}

Shi-Kui Zhao, born in 1984, is currently an associate professor at School of Mechanical Engineering, University of Jinan, China. He received his $\mathrm{PhD}$ degree from Zhejiang Universtiy, China, in 2013. His research interests include shop scheduling and intelligent scheduling optimization.

Tel: +86-531-89736314; E-mail: me_zhaosk@ujn.edu.cn

Lin Huang, born in 1995, is currently a master candidate at School of Mechanical Engineering, University of Jinan, China.

Qing Han, born in 1982, is currently an associate professor at School of Mechanical Engineering, University of Jinan, China. She received her PhD degree from Zhejiang Universtiy, China, in 2012.

Corresponding author: Shi-Kui Zhao E-mail: me_zhaosk@ujn.edu.cn 


\title{
A Hybrid Iterated Greedy Algorithm for Job Shop Scheduling Problem
}

\author{
Shi-Kui Zhao • Lin Huang • Qing Han
}

Received June xx, 201x; revised February xx, 201x; accepted March xx, 201x

(C) Chinese Mechanical Engineering Society and Springer-Verlag Berlin Heidelberg 2017

\begin{abstract}
Aiming at the job shop scheduling problem (JSP), an iterated greedy hybrid algorithm is proposed to optimize the maximum completion time. In the sections of deconstruction and reconstruction of iterated greedy algorithm, any number of operations can be moved to realize the deconstruction and reconstruction of operation-level. When deconstructing, after removing multiple operations, the calculation method of the head and tail length of the partial scheduling based on adjacency matrix and topological sorting is studied. The corresponding directed graph model and adjacency matrix are updated, to solve the topological sorting based on the adjacency matrix, and then the head and tail length of the operations in the partial scheduling are calculated. In the reconstruction, multiple operations should be inserted into the existing partial scheduling. The reconstruction method is based on the insertion algorithm of constructing the initial scheduling, and each previously removed operation should be inserted in turn, which not only ensures the feasibility after inserting multiple operations, but also scientifically inserts operations with better strategies. In view of the strong local search ability of tabu search algorithm, the tabu search is used in the local search section of iterated greedy algorithm. The effectiveness of the proposed algorithm is verified by testing international JSP benchmarks.
\end{abstract}

Keywords: Job shop scheduling problem • Tabu search • Iterated greedy algorithm - Deconstruction and reconstruction • Maximum completion time

\section{Introduction}

Job shop scheduling problem (JSP) is an abstract model of scheduling problems in many manufacturing enterprises

Shi-Kui Zhao

me_zhaosk@ujn.edu.cn

School of Mechanical Engineering, University of Jinan, Jinan 250022, China and service industries, and its research has important scientific theoretical significance and practical application value.

Intelligent algorithm is an effective solution to JSP, which can calculate a better solution in a feasible time. Among many intelligent algorithms, tabu search algorithm has been widely used in the design of single algorithm and hybrid algorithm, and shows excellent performance. Taillard [1] successfully applied tabu search algorithm to solve JSP for the fist time. Nowicki et al. [2] proposed a tabu search algorithm (TSAB) based on critical path and block structure to solve JSP in 1996. On this basis, they adopted the initial solution generation and search method based on path relinking and proposed a more efficient $i$-TSAB algorithm [3] in 2002. In 2007, Zhang et al. [4] proposed a tabu search algorithm based on N7 neighborhood structure by expanding the operation movement range of neighborhood structure, and combined tabu search with simulated annealing algorithm [5] in 2008 to further improve the performance of JSP solving algorithm. In 2012, Nasiri et al. [6] used the new N6 neighborhood structure to solve JSP by combining global equilibrium search and tabu search algorithm, and improved the current best solution of 19 benchmark instances of JSP. In 2014, Gonçalves et al. [7] combined random key genetic algorithm with tabu search algorithm, designed mobile job search technology based on diagram model, and improved the best solution of 57 benchmark instances of JSP. In 2015, Peng et al. [8] combined path relinking and tabu search algorithm based on N7 neighborhood structure to solve JSP, which improved the best solution of the current 49 benchmark instances of JSP. In 2016, Cheng et al. [9] designed a hybrid evolutionary algorithm integrating tabu search algorithm to solve JSP. In 2018, Nagata et al. [10] mixed tabu search and pop-up 
bottleneck operation algorithm to solve JSP. In 2021, Mahmud et al. [11] proposed a hybrid algorithm of differential evolution and tabu search to solve JSP, and Zhao [12] designed a hybrid algorithm of path relinking and tabu search, both of which achieved good results. In addition, tabu search algorithm has also been successfully applied to flexible job shop scheduling problem (FJSP), such as advanced FJSP algorithms proposed by Mastrolilli [13], González [14], Ding [15], Li [16] et al. These studies show the effectiveness of tabu search algorithm. Tabu search algorithm can be well integrated with the neighborhood structure of the problem. At the same time, it is found that in order to better compensate for the global search performance of tabu search algorithm, other evacuation search mechanisms are usually integrated, such as path relinking, hybrid algorithms and so on.

As a simple and effective algorithm, iterated greedy algorithm has been widely used in job shop scheduling problems in recent years. The algorithm first initializes a solution, and then improves the current solution through deconstruction, reconstruction and local search. Ruiz et al. [17] first applied the iterated greedy algorithm to solve the permutation flow shop scheduling problem. Minella et al. [18] solved multi-objective flow shop scheduling problem based on iterated greedy algorithm. Pan et al. [19] used iterated greedy algorithm to solve the problem of no-idle permutation flow shop. Tasgetiren et al. [20] applied the iterated greedy algorithm to solve the blocking flow shop scheduling problem. Shao et al. [21] studied the iterated greedy algorithm for distributed no-wait flow shop scheduling problem. Ruiz et al. [22] studied the iterated greedy algorithm for solving the distributed permutation flow shop scheduling problem. For job shop scheduling problem, Deng et al. [23] studied the no-wait job shop scheduling problem based on the population-based iterated greedy algorithm with the total flow time as the goal. Pranzo et al. [24] used the iterated greedy algorithm to solve the blocking job shop scheduling problem. Kim et al. [25] used iterated greedy algorithm to solve job shop scheduling problem with job family and sequence related setup time. Aqel et al. [26] applied the iterated greedy algorithm to solve the flexible job shop scheduling problem. Thus, the iterated greedy algorithm has been widely used in the flow shop scheduling problem, and in the process of deconstruction and reconstruction, the reconstruction mechanism based on the $\mathrm{NEH}$ (Nawaz-Enscore-Ham) algorithm [27] is usually adopted. However, there are few applications in JSP, and there is still a lack of effective deconstruction and reconstruction mechanism, especially the iterated greedy algorithm aiming at maximum completion time.

Integrating the good global search ability of iterated greedy algorithm in deconstruction and reconstruction, and the concentrated search ability of tabu search algorithm, the two are mixed to solve JSP. At the same time, in the process of deconstruction and reconstruction of the iterated greedy algorithm, a deconstruction and reconstruction mechanism that can arbitrarily move multiple operations is designed, which not only ensures the feasibility of the solution after inserting multiple operations, but also carries out scientific insertion operations with a better strategy. A hybrid algorithm of iterated greedy and tabu search is designed and implemented, and the effectiveness of the proposed algorithm is verified by testing international JSP benchmarks. The remainder of this paper is arranged as follows. Problem description is presented in Section 2. Section 3 discusses the deconstruction and reconstruction mechanism. Section 4 discusses the hybrid iterated greedy algorithm. Experimental studies are discussed in Section 5. Section 6 provides the conclusions and future work.

\section{Problem Description}

The JSP can be described as that $n$ jobs are processed on $m$ machines, the job set is $J$, the machine set is $M$, and the number of operations of job $J_{i}$ is $o_{i}$. According to the process route, the operations numbers of job $J_{i}$ are $\left(l_{i-1}+\right.$ $\left.1, \ldots, l_{i-1}+o_{i}\right)$, Where $l_{i-1}$ is the sum of the operation numbers of the previous $i-1$ jobs, and the operations set of all jobs is $O$. For an operation $x$, the processing machine is $u_{x}$, the corresponding processing time is $p_{x}$, the previous operation and subsequent operation of the job are $J P[x]$ and $J S[x]$ respectively, and the previous operation and subsequent operation of the machine are $M P[x]$ and $M S[x]$ respectively. The operation set $O$ can be divided into multiple subsets $M_{k}=\left\{i \in O: \mu_{i}=k\right\}$ according to the processing machine, and the operations processing sequence on machine $k$ can be expressed as $\pi(k)=$ $\left(\pi_{k}(1), \ldots, \pi_{k}\left(\left|M_{k}\right|\right)\right)$, where $\pi_{k}(i)$ represents the operation at the $i$ th position in $\pi(k)$, and the operation processing sequence on all machines can be represented as a scheduling scheme $\pi=\left(\pi_{1}, \ldots, \pi_{m}\right)$. In order to facilitate the subsequent description of the method in this paper, some of the above symbol definitions refer to the format in Ref. [3]. The rest of the assumptions are as follows:

(1) A job can only be processed simultaneously on one machine at most.

(2) Each machine can process at most one operation at a time. 
(3) There are sequence constraints between operations of the same job.

(4) Once an operation initiates processing on a given machine, it must complete processing on that machine without interruption.

The objective of this paper is to optimize the maximum completion time $\left(C_{\max }\right)$, and the objective function is expressed as Eq. (1), where $C_{i}$ is the completion time of the job $J_{i}$.

$$
C_{\max }=\min \left(\max _{1 \leq i \leq n} C_{i}\right),
$$

Table 1 shows a $4 \times 4$ JSP example, including 4 jobs and 4 machines, with a total of 16 operations. Figure 1 shows a scheduling Gantt chart of the example, with a corresponding maximum completion time of 51 . The subsequent methods are described with the given example.
Table 1 A $4 \times 4$ JSP example

\begin{tabular}{ccccc}
\hline Job & Operation & $\begin{array}{c}\text { Operation } \\
\text { number }\end{array}$ & Machine & Processing time \\
\hline J1 & 1,1 & 1 & M3 & 1 \\
& 1,2 & 2 & M1 & 3 \\
& 1,3 & 3 & M2 & 6 \\
& 1,4 & 4 & M4 & 7 \\
\hline J2 & 2,1 & 5 & M2 & 8 \\
& 2,2 & 6 & M3 & 5 \\
& 2,3 & 7 & M4 & 10 \\
& 2,4 & 8 & M1 & 10 \\
\hline J3 & 3,1 & 9 & M3 & 5 \\
& 3,2 & 10 & M4 & 4 \\
& 3,3 & 11 & M2 & 8 \\
& 3,4 & 12 & M1 & 9 \\
\hline J4 & 4,1 & 13 & M2 & 5 \\
& 4,2 & 14 & M1 & 5 \\
& 4,3 & 15 & M3 & 5 \\
& 4,4 & 16 & M4 & 3 \\
\hline
\end{tabular}

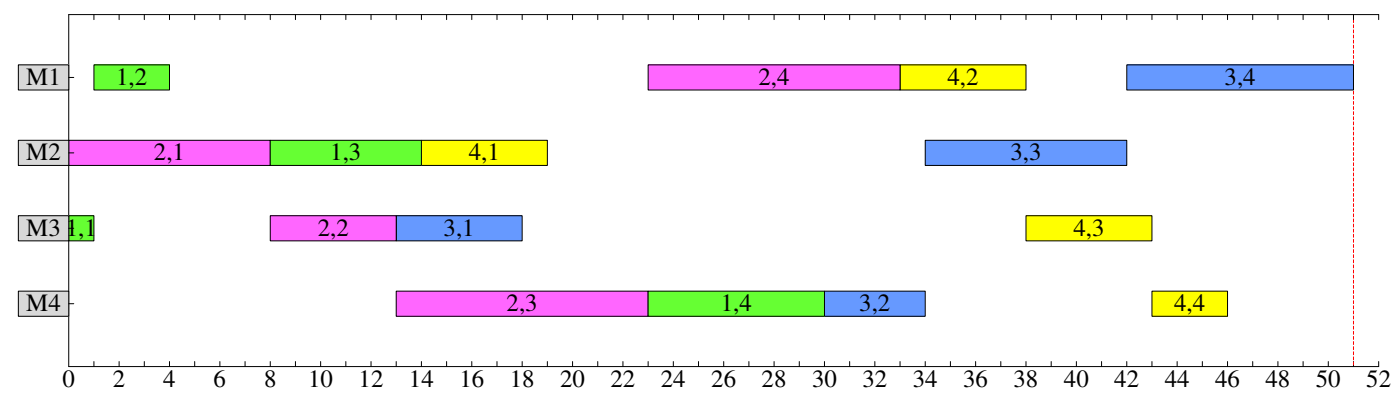

Figure 1 Gantt chart of a $4 \times 4$ JSP example $\left(C_{\max }=51\right)$

\section{Deconstruction and Reconstruction}

Deconstruction and reconstruction are important parts of iterated greedy algorithm, which is of great significance for iterated greedy algorithm to escape from local optimum, avoid premature convergence and realize more global decentralized search. In the existing iterated greedy algorithm to solve JSP problem, it is more for the deconstruction and reconstruction of job-level. To optimize makespan as the goal, the realization of arbitrary multiple operation-level of deconstruction and reconstruction, for solving the JSP problem, have important research value. Given a JSP scheduling solution, firstly, multiple operations need to be removed by deconstruction to obtain a partial scheduling, and then, through reconstruction, the removed multiple operations are inserted into the partial scheduling with a certain strategy, so as to construct a new complete scheduling. During deconstruction, the partial schedules need to be updated to prepare for reconstruction.
In the process of reconstruction, multiple operations should be inserted into the existing partial scheduling. How to ensure the feasibility of the solution after multiple operations are inserted and insert operations scientifically with better strategies are the problems to be solved in reconstruction. Therefore, this paper designs an operation-level deconstruction and reconstruction mechanism based on the insertion algorithm of JSP problem construction initial scheduling.

\subsection{Insertion Algorithm}

At present, there are many studies on iterated greedy algorithm for solving flow shop scheduling problems. In the process of deconstruction and reconstruction, NEH algorithm [27] is used. The principle of NEH scheduling is determined according to the descending order of the sum of processing time of each job on all machines. In the job shop scheduling problem, Nowicki and Smutnicki proposed an insertion algorithm (INSA) [3] to construct 
initial solutions, which returned a feasible scheduling solution, that is, the operations processing sequence $\sigma=\left(\sigma_{1}, \sigma_{2}, \ldots, \sigma_{m}\right)$ on each machine. In the famous TSAB algorithm [2] and $i$-TSAB algorithm [3], the construction of the initial solution is realized by the INSA. The specific implementation steps of the INSA are as follows:

Step 1: Calculate the sum of all operations processing time of each job, find out the job with the largest sum of operations processing time, schedule all operations $\left\{1, \ldots, l_{1}\right\}$ of the job, so as to construct the initial partial scheduling $\sigma$.

Step 2: All operations $\left\{l_{1}+1, l_{1}+2, \ldots, o\right\}$ of the remaining jobs are sorted in descending order according to the processing time, and the sequence $w$ is obtained, to schedule the remaining operations according to the sequence $w$.

Step 3: For operations $s=1$ to $o-l_{1}$ in sequence $w$, perform step 4 .

Step 4: For a partial scheduling $\sigma$, operation $x=w(s)$, the processing machine is $a=u_{x}$, and $\left|\sigma_{a}\right|$ is the number of existing operations on machine $a$. Therefore, there are $z=\left|\sigma_{a}\right|+1$ insertion positions of operation $x$, and $z$ scheduling schemes $\sigma^{j}$ can be obtained accordingly, and each scheduling scheme satisfies $\sigma_{k}^{j}=\sigma_{k}, k \neq a$ and

$$
\sigma_{a}^{j}=\left(\sigma_{a}(1), \ldots, \sigma_{a}(j-1), x, \sigma_{a}(j) \ldots, \sigma_{a}\left(\left|\sigma_{a}\right|\right)\right),
$$

Where $j=1, \ldots, z$, find the optimal scheduling $\sigma^{\prime}$, and satisfy

$$
L_{x}=\max \left\{r_{J P[x]}, r_{\sigma_{a}(j-1)}\right\}+p_{x}+\max \left\{q_{J S[x]}, q_{\sigma_{a}(j)}\right\}
$$

minimum, in Eq. (3), $L_{x}$ indicates the longest path through operation $x$ in the directed graph corresponding to scheduling $\sigma^{\prime}$ after inserting the operation, and satisfies the condition that the directed graph in $\sigma^{\prime}$ does not contain a directed circuit, and then set $\sigma=\sigma^{\prime}$.

\subsection{Deconstruction Mechanism}

During deconstruction, multiple operations need to be removed from the current schedule to obtain a partial schedule. For this partial schedule, relevant data information needs to be updated to prepare for the reconstruction of insertion operations. Taking the scheduling Gantt diagram shown in Figure 1 as an example, the operations processing sequence of each machine is shown in Figure 2(a), and the corresponding directed graph model of operations processing sequence is shown in Figure 2(b) according to the permutation-graph model in Ref. [3]. In the job dimension, it corresponds to the process route sequence of each job, which is connected by the connecting arc arrow; in the machine dimension, it corresponds to the process processing sequence of each machine, which is connected by the disjunctive arc arrow. When there is no directed loop in the directed graph, it corresponds to a feasible solution. Otherwise, it is an infeasible solution. The permutation-graph model of JSP is different from the disjunctive graph model in that there are no virtual start operation node and end operation node.

Deconstruction needs to remove multiple operations at once, and after removing the operations, a partial scheduling is obtained. At this time, the corresponding directed graph model needs to be updated, and more importantly, the length of the head and tail of the operations needs to be updated, so that the previously removed operations can be reasonably inserted through calculation. Therefore, the head and tail length updating method of partial scheduling operations based on adjacency matrix is studied. Taking the scheduling shown in Figure 1 as an example, after removing operations $(1,4$, 2,2, 2,3, 2,4, 4,3) (the corresponding operations numbers are $4,6,7,8,15)$, the obtained partial scheduling digraph model is shown in Figure 3. In the directed graph, the connecting arc representing the process route sequence of the job remain operations unchanged, while the disjunctive arc representing the process sequence on each machine needs to be updated. In Figure 3 , arcs $2 \rightarrow 8$, $8 \rightarrow 14,1 \rightarrow 6,6 \rightarrow 9,7 \rightarrow 4,4 \rightarrow 10$, and $9 \rightarrow 15$ need to be deleted, and arcs $2 \rightarrow 14$ and $1 \rightarrow 9$ need to be added. The change of the corresponding adjacency matrix before and after removing the operations is shown in Figure 4. The dimension of the adjacency matrix is equal to the sum of the number of all the operations, which is convenient for concise description and eliminates 0 in the matrix. In the adjacency matrix, the deleted element 1 corresponds to the deleted arc in Figure 3, and the newly added element 1 corresponds to the newly added arc in Figure 3. According to the adjacency matrix corresponding to the partial scheduling in Figure 4(b), the topological sequence is $(5,6,7,8,1,9,10,2,3,13,14,15,16,11,12$ and 4). According to the sequence, the head and tail lengths of the operations are calculated forward and backward respectively to obtain the partial scheduling Gantt chart as shown in Figure 5. The dotted box in Figure 5 indicates the removed operations. Figure 5(a) is the Gantt chart of the earliest start and completion time of the operations, corresponding to the length of the operations head, and 
Figure 5(b) is the Gantt chart of the latest start and completion time of the operations, corresponding to the length of the operations tail.

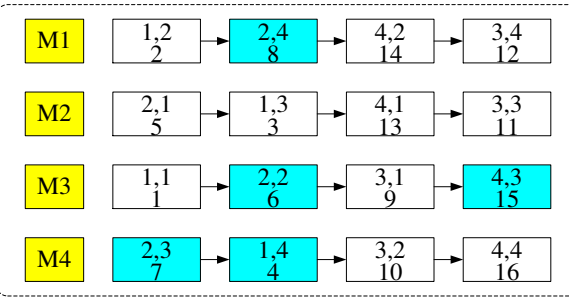

(a) Operations sequence of each machine

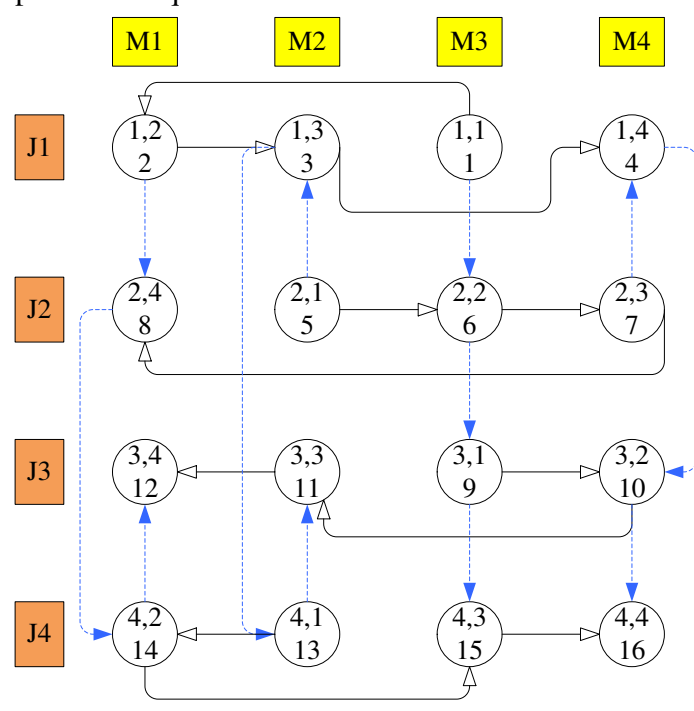

(b) Directed graph corresponding to operations sequence

Figure 2 Operations processing sequence and directed graph model

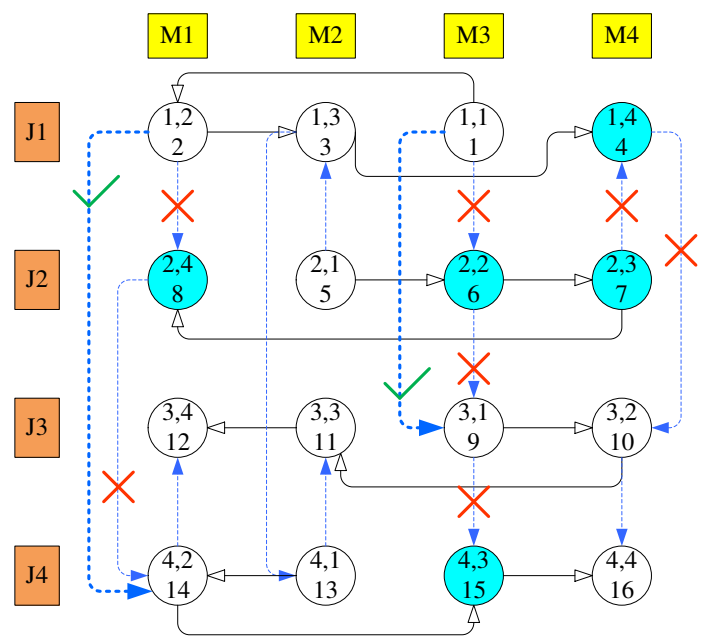

Figure 3 Directed graph model of partial scheduling after removing operations
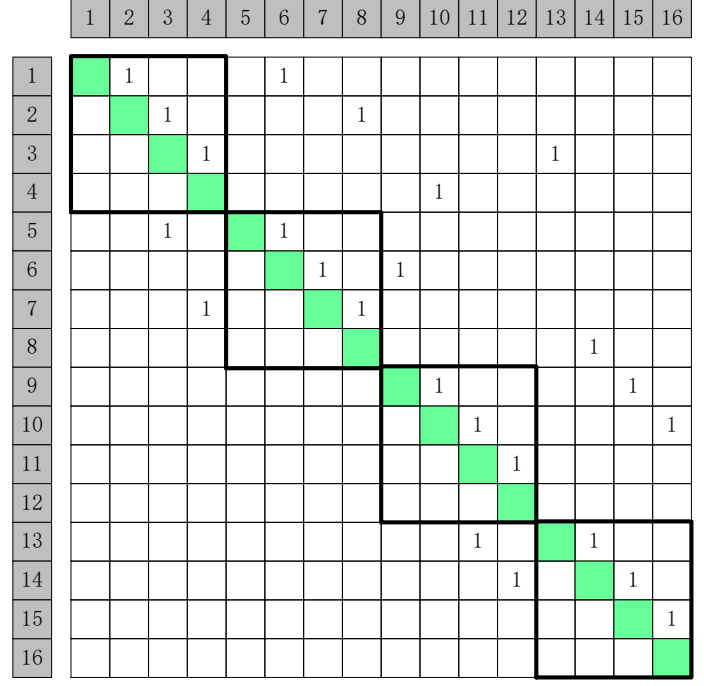

(a) Adjacency matrix of the original scheduling

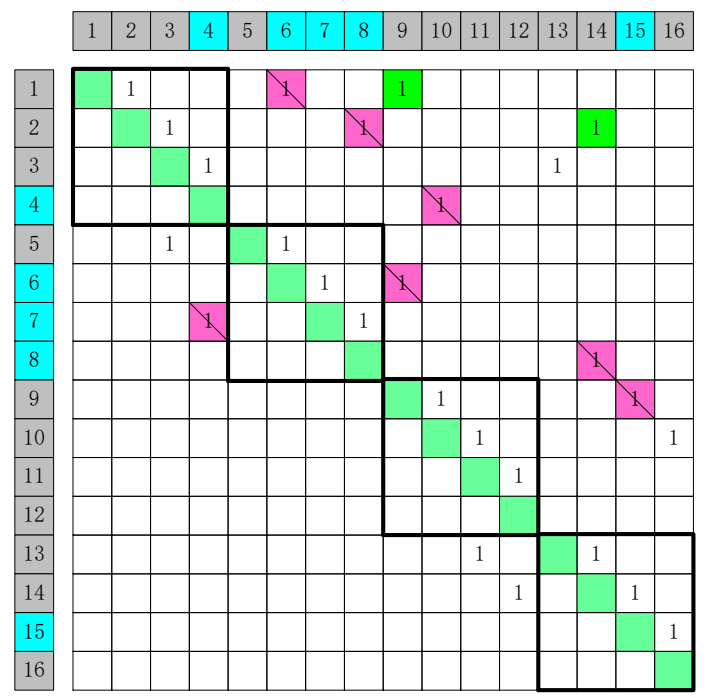

(b) Adjacency matrix of partial scheduling after removing operations

Figure 4 Adjacency matrix before and after removing operations

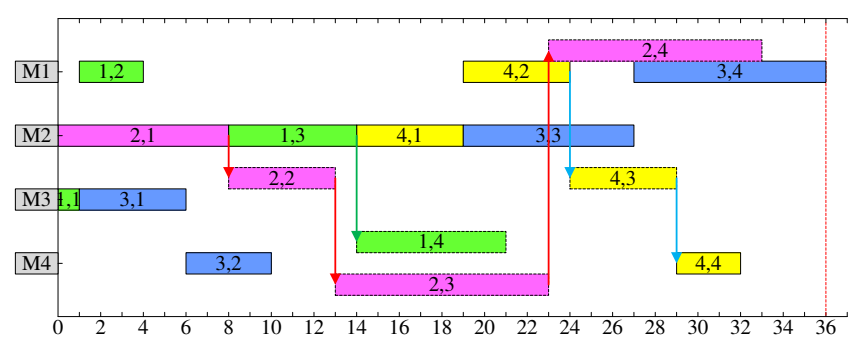

(a) Gantt chart of the earliest start and finish time of partial scheduling operations 


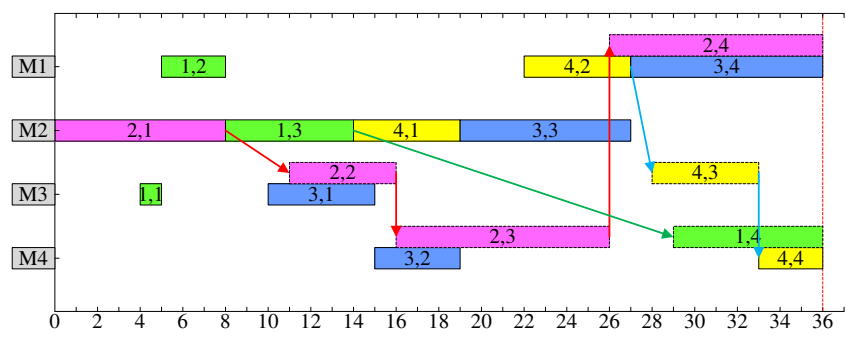

(b) Gantt chart of the latest start and finish time of partial scheduling operations

Figure 5 Gantt chart of partial scheduling (dotted box indicates removed operations)

\subsection{Reconstruction Mechanism}

During reconstruction, multiple operations should be inserted into the existing partial scheduling, which not only ensures the feasibility of the new solution after inserting multiple operations, but also scientifically inserts operation with better strategies. The insertion method based on INSA [3] is designed, and each previously removed operation is inserted in turn. Figure 6 shows the analysis of the insertion position of the operation $x$. For a partial scheduling $\sigma$, the corresponding directed graph is $G(\sigma)$. When inserting the operation $x$, the processing machine is $a=u_{x}$, and the operations processing sequence on machine $a$ is $\sigma_{a}=\left(\sigma_{a}(1), \sigma_{a}(2) \ldots, \sigma_{a}\left(\left|\sigma_{a}\right|\right)\right)$, there are $z=\left|\sigma_{a}\right|+1$ insertion positions of operation $x$, and $z$ scheduling schemes are obtained accordingly. Ensuring the optimal and feasible insertion position is the key. As shown in Figure 6 , in sequence $\sigma_{a}, e$ is the maximum position where the directed path $\sigma_{a}(e) \rightarrow x$ exists. If the directed path does not exist, then $e=0$. The $f$ is the minimum position where the directed path $x \rightarrow \sigma_{a}(f)$ exists. If the directed path does not exist, then $f=\left|\sigma_{a}\right|+1$. Since the operation $x$ has not been inserted into machine $a$, if the directed path $\sigma_{a}(e) \rightarrow x$ exists, the required point is $J P[x]$, which is in the form of $\sigma_{a}(e) \rightarrow J P[x] \rightarrow x$; if the directed path $x \rightarrow \sigma_{a}(f)$ exists, the required point is $J S[x]$, which is in the form of $x \rightarrow J S[x] \rightarrow \sigma_{a}(f)$.

In terms of the optimality of the insertion position, the longest path through the operation $x$ after the insertion operation $x$ is calculated as shown in Eq. (3). In a directed graph, the head length of an operation node is the length of the longest path to the operation, including the processing time of the operation, while the tail length is the length of the longest path from the operation, including the processing time of the operation. The insertion position $h$ within the insertion range $[1, e]$ satisfies $1 \leq h \leq e$, due to the existence of a directed path $\sigma_{a}(e) \rightarrow J P[x] \rightarrow x$, so $r_{J P[x]}>r_{\sigma_{a}(e)}$. Because there are directed paths $\sigma_{a}(h) \rightarrow \sigma_{a}(e+1)$ and $\sigma_{a}(h) \rightarrow J S[x], \quad$ it satisfies $q_{\sigma_{a}(h)}>q_{\sigma_{a}(e+1)}$ and $q_{\sigma_{a}(h)}>q_{J S[x]}$, that is $q_{\sigma_{a}(h)}>\max \left\{q_{J S[x]}, q_{\sigma_{a}(e+1)}\right\}$. Therefore, at the insertion position $h$, the longest path through the operation $x$ has the following properties:

$$
\begin{aligned}
L_{x}(h) & =\max \left\{r_{J P[x]}, r_{\sigma_{a}(h-1)}\right\}+p_{x}+\max \left\{q_{J S[x]}, q_{\sigma_{a}(h)}\right\} \\
& \geq r_{J P[x]}+p_{x}+q_{\sigma_{a}(h)} \\
& >\max \left\{r_{J P[x]}, r_{\sigma_{a}(e)}\right\}+p_{x}+\max \left\{q_{J S[x]}, q_{\sigma_{a}(e+1)}\right\} \\
& =L_{x}(e+1) .
\end{aligned}
$$

The insertion position $h$ within the insertion range $\left[f+1,\left|\sigma_{a}\right|+1\right]$ satisfies $f+1 \leq h \leq\left|\sigma_{a}\right|+1$. Since there is a directed path $x \rightarrow J S[x] \rightarrow \sigma_{a}(f)$, so $q_{J S[x]}>q_{\sigma_{a}(f)}$. Since there are directed paths $\sigma_{a}(f-$ 1) $\rightarrow \sigma_{a}(h-1)$ and $J P[x] \rightarrow \sigma_{a}(h-1), \quad r_{\sigma_{a}(h-1)}>$ $r_{\sigma_{a}(f-1)}$ and $r_{\sigma_{a}(h-1)}>r_{J P[x]}$ are satisfied, that is $r_{\sigma_{a}(h-1)}>\max \left\{r_{J P[x]}, r_{\sigma_{a}(f-1)}\right\}$. Therefore, at the insertion position $h$, the longest path through the operation $x$ has the following properties:

$$
\begin{aligned}
L_{x}(h) & =\max \left\{r_{J P[x]}, r_{\sigma_{a}(h-1)}\right\}+p_{x}+\max \left\{q_{J S[x]}, q_{\sigma_{a}(h)}\right\} \\
& \geq r_{\sigma_{a}(h-1)}+p_{x}+q_{J S[x]} \\
& >\max \left\{r_{J P[x]}, r_{\sigma_{a}(f-1)}\right\}+p_{x}+\max \left\{q_{J S[x]}, q_{\sigma_{a}(f)}\right\} \\
& =L_{x}(f) .
\end{aligned}
$$

It can be seen the longest path through operation $x$ is greater than the insertion point $e+1$ within the insertion range $[1, e]$. The insertion range $\left[f+1,\left|\sigma_{a}\right|+1\right]$ is larger than the insertion point $f$. Therefore, the optimal insertion range is $[e+1, f]$.

In terms of the feasibility of insertion position, due to the existence of directed path $\sigma_{a}(e) \rightarrow x$, inserting the operation $x$ in position range $[1, e]$ will inevitably produce directed path $x \rightarrow \sigma_{a}(e)$, leading to the generation of directed loop $\sigma_{a}(e) \rightarrow x \rightarrow \sigma_{a}(e)$. Therefore, a feasible solution cannot be obtained within the insertion position range $[1, e]$. Similarly, due to the existence of directed path $x \rightarrow \sigma_{a}(f)$, the feasible solution of insertion position range $\left[f+1,\left|\sigma_{a}\right|+1\right]$ cannot be obtained. It can be seen the operation insertion position range to ensure the feasible solution is $[e+1, f]$. Therefore, as long as Eq. (3) is minimized and the longest path formula through operation $x$ is minimized, the optimality of the insertion position and the feasibility of the insertion position are guaranteed. In addition, in the 
process of constructing the initial solution, the operations are sorted in descending order according to the processing time of the operations, and each operation is inserted in turn, while in the reconstruction process, each operation is inserted in random order, so as to achieve a more free and decentralized reconstruction. In particular, for the classic JSP problem, each job is processed once on all machines, in the process of constructing the initial solution using INSA method [3], because a job has been scheduled, the number of existing processing operations of the machine is at least 1 . However, in the reconstruction process after deconstruction, because multiple operations are arbitrarily removed, it is possible to insert an operation into the machine, and the number of existing processes of the machine is 0 . At this time, insert the operation into the machine for processing, and no new disjunctive arc will be added to the corresponding directed graph. Because the existing partial scheduling is feasible and there is no directed loop in the directed graph, the feasibility of the post insertion scheduling scheme is guaranteed. The Gantt chart corresponding to the partial scheduling is shown in Figure 5, after the removed operations are inserted again, the reconstructed scheduling Gantt chart is shown in Figure 7, and the maximum completion time is $C_{\max }=43$.

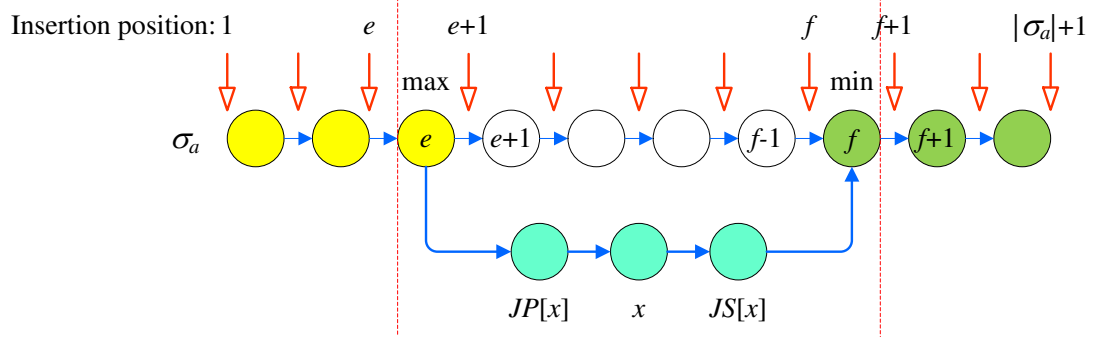

Figure 6 Analysis of the insertion position of operation $x$.

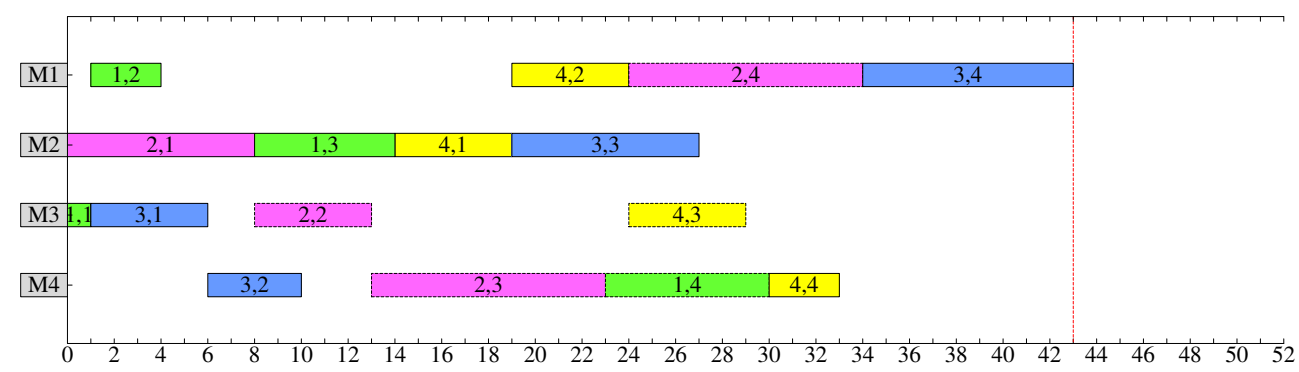

Figure 7 Reconstructed scheduling Gantt chart $\left(C_{\max }=43\right)$

\section{Hybrid Iterated Greedy Algorithm}

Iterated greedy algorithm is a simple and effective iterated search algorithm. First initialize a solution, and then continuously improve the current solution by deconstructing, reconstructing, local search, and use the greedy strategy to accept better new solutions. Otherwise, the reference to the principle of simulated annealing algorithm to accept difference solution with a certain probability, as shown in Eqs. (4), (5), $\operatorname{sum}\left(p_{i}\right)$ is the sum of the processing time of all operations. In the iterated greedy algorithm, the large-scale perturbation of the current solution is realized through deconstruction and reconstruction, and then the global search is realized. With the help of local search operator, the algorithm has both global and local search capability.

$$
\begin{gathered}
\text { random } \leq \exp \{-(\text { Snew.Cmax }- \text { Sc.Cmax }) / \text { temp }\},(4) \\
\text { temp }=0.4 \cdot \operatorname{sum}\left(p_{i}\right) /(n \cdot m \cdot 10),
\end{gathered}
$$

In view of the strong centralized local search ability of tabu search algorithm, tabu search algorithm is used in the local search of iterated greedy algorithm, and then iterated greedy hybrid algorithm is realized. Tabu search algorithm is a sub heuristic random search algorithm. It starts from an initial feasible solution and generates multiple neighborhood solutions based on the neighborhood operation defined by the neighborhood structure, and then realizes heuristic search around the current solution. In order to avoid falling into local optimization, Tabu search uses a flexible memory technology to record the searched solutions to guide the 
next search direction, which is the establishment of tabu list. In this paper, the neighborhood structure in Ref. [28] is used to generate the neighborhood solution, and the fast approximate evaluation method in Ref. [29] is used to evaluate the neighborhood solution. For the neighborhood solution, the solution whose evaluation value is better than the historical optimal solution and meets the amnesty criterion is preferred. Secondly, non tabu solutions with better evaluation value are selected. Otherwise, if all the neighborhood solutions are in tabu state, the better tabu solution is selected. Tabu list uses the operation sequence and specific position method in Ref. [4] to record part of the attributes of the new solution, so as to avoid the algorithm's devious search returning to the previously searched historical solution and falling into local optimum. The dynamic tabu length setting mode is adopted, the tabu length is dynamically selected between the two extreme values $L_{\min }$ and $L_{\max }$. The calculation formula is as follows, where ceil represents an up-rounding function and returns the minimum integer greater than or equal to the specified expression value, $n$ and $m$ are the number of jobs and machines respectively.

$$
\begin{aligned}
& L_{\text {min }}=\operatorname{ceil}(0.5 \cdot(10+n / m)), \\
& L_{\text {max }}=\operatorname{ceil}(1.5 \cdot(10+n / m)),
\end{aligned}
$$

\section{Experiments and Discussion}

In order to test the performance of the iterated greedy hybrid algorithm designed in this paper, the international benchmark instance of JSP problem is used to test. The proposed algorithm is implemented in MATLAB on a personal computer with $3.20 \mathrm{GHz} \mathrm{CPU}$ and $8.0 \mathrm{~GB}$ memory.

After many tests, the algorithm parameters are set as follows: the maximum number of iterations of the iterated greedy algorithm is 100, the number of removed operations is 10 during deconstruction, and the maximum number of iterations of tabu search algorithm is 6000 . The international benchmark instances of JSP problem, including FT06, FT10, FT20, LA01 LA40, ORB01 ORB10, a total of 53 instances, and each instance is solved 20 times. In order to compare the performance of different algorithms, the test results are compared with those of other relevant algorithms in references.

Table 2 shows the comparison between the test results of LA and FT benchmark instances of JSP problems. In Table $2, C_{\max }$ is the optimal value of the solution result for 20 times for each instance. $A V\left(C_{\max }\right)$ is the average of the solution result for 20 times. $A V(\mathrm{CPU})$ is the average time to obtain the optimal value $C_{\max }$, in seconds. $R E$ is the relative deviation between the optimal value of the solution result and the lower bound $L B$ of the optimal solution. The five comparison algorithms in Table 2 include parallel artificial bee colony algorithm (pABC) in Ref. [30], biased random-key genetic algorithm (BRKGA) in Ref. [7], differential evolution and tabu search hybrid algorithm (MCDE/TS) in Ref. [11], hybrid genetic algorithm (HGA) in Ref. [31], path relinking and tabu search hybrid algorithm (PRTS) in Ref. [12]. Statistical comparative analysis is conducted from the perspective of relative deviation, to calculate the relative deviation $R E$ between $C_{\max }$ value and the lower limit $L B$. The calculation of $R E$ is shown in Eq. (8).

$$
R E=\left(C_{\max }-L B\right) / L B \times 100,
$$

The average $M R E$ is obtained for the relative deviation of 43 JSP problems. The $M R E$ of the proposed algorithm is 0.002 , and the $M R E$ of the other five algorithms are $0.023,0.002,0.312,0.176$ and 0.012 respectively. The solution result of the proposed algorithm is better than four of them, which is equivalent to that of random key genetic algorithm (BRKGA). As the pABC algorithm does not provide the LA36-LA40 test results, the relative deviation $R E$ of the five benchmark examples is set to 0 when calculating the mean $M R E$ of the relative deviation. Among the 43 benchmark instances, this paper algorithm obtains the lower bound of the other 42 benchmark instances except LA29. LA29 and LA40 are the most complex instances of LA. The $C_{\max }$ value of LA29 obtained by the proposed algorithm is 1153, which is better than four algorithms. The $C_{\max }$ value of the LA40 instance obtained by the algorithm in this paper is 1222 , which is the optimal solution of the instance, and better than three algorithms, which verifies the effectiveness of the proposed algorithm. Figures 8 and 9 are Gantt charts of the scheduling results of LA29 and LA40 benchmark instances obtained by the proposed algorithm respectively.

Table 3 shows the comparison between the test results of the algorithm in this paper and the results of other algorithms for the ORB benchmark instances. The five comparison algorithms in Table 3 include the tabu search and simulated annealing hybrid algorithm (TS/SA) in Ref. [5], artificial bee colony algorithm (BeFABC) in Ref. [31], hybrid particle swarm optimization and variable neighborhood search algorithm (HPV) in Ref. [33], tabu search and path relinking hybrid algorithm (TS/PR) in Ref. [5], And biased random key genetic algorithm (BRKGA) 
in Ref. [7]. Statistical comparative analysis is carried out from the perspective of relative deviation from the lower bound value LB of the instance. The algorithm in this paper obtains the lower bound value for 10 ORB benchmark instances, and the $M R E$ is 0 , which achieves the same solution result as the five comparison algorithms, and then verifies the effectiveness of the algorithm in this paper.

Table 2 Computational results and comparisons of LA and FT benchmark instances

\begin{tabular}{|c|c|c|c|c|c|c|c|c|c|c|c|c|c|c|c|c|}
\hline \multirow[t]{2}{*}{ Instance } & \multirow[t]{2}{*}{$n \times m$} & \multirow[t]{2}{*}{$L B$} & \multicolumn{4}{|c|}{ Proposed algorithm } & \multicolumn{2}{|c|}{$\begin{array}{c}\mathrm{pABC} \\
{[30]}\end{array}$} & \multicolumn{2}{|c|}{$\begin{array}{c}\text { BRKGA } \\
{[7]} \\
\end{array}$} & \multicolumn{2}{|c|}{$\begin{array}{c}\mathrm{MCDE} / \mathrm{TS} \\
{[11]} \\
\end{array}$} & \multicolumn{2}{|c|}{$\begin{array}{c}\text { HGA } \\
{[31]}\end{array}$} & \multicolumn{2}{|c|}{$\begin{array}{c}\text { PRTS } \\
{[12]}\end{array}$} \\
\hline & & & $C_{\max }$ & $A V\left(C_{\max }\right)$ & $A V(\mathrm{CPU})$ & $R E$ & $C_{\max }$ & $R E$ & $C_{\max }$ & $R E$ & $C_{\max }$ & $R E$ & $C_{\max }$ & $R E$ & $C_{\max }$ & $R E$ \\
\hline LA01 & $10 \times 5$ & 666 & 666 & 666 & 0.05 & 0 & 666 & 0.00 & 666 & 0 & 666 & 0 & 666 & 0.00 & 666 & 0 \\
\hline LA02 & $10 \times 5$ & 655 & 655 & 655 & 0.77 & 0 & 655 & 0.00 & 655 & 0 & 655 & 0 & 655 & 0.00 & 655 & 0 \\
\hline LA03 & $10 \times 5$ & 597 & 597 & 597 & 2.26 & 0 & 597 & 0.00 & 597 & 0 & 597 & 0 & 597 & 0.00 & 597 & 0 \\
\hline LA04 & $10 \times 5$ & 590 & 590 & 590 & 0.4 & 0 & 590 & 0.00 & 590 & 0 & 590 & 0 & 590 & 0.00 & 590 & 0 \\
\hline LA05 & $10 \times 5$ & 593 & 593 & 593 & 0.01 & 0 & 593 & 0.00 & 593 & 0 & 593 & 0 & 593 & 0.00 & 593 & 0 \\
\hline LA06 & $15 \times 5$ & 926 & 926 & 926 & 0.04 & 0 & 926 & 0.00 & 926 & 0 & 926 & 0 & 926 & 0.00 & 926 & 0 \\
\hline LA07 & $15 \times 5$ & 890 & 890 & 890 & 0.1 & 0 & 890 & 0.00 & 890 & 0 & 890 & 0 & 890 & 0.00 & 890 & 0 \\
\hline LA08 & $15 \times 5$ & 863 & 863 & 863 & 0.06 & 0 & 863 & 0.00 & 863 & 0 & 863 & 0 & 863 & 0.00 & 863 & 0 \\
\hline LA09 & $15 \times 5$ & 951 & 951 & 951 & 0.04 & 0 & 951 & 0.00 & 951 & 0 & 951 & 0 & 951 & 0.00 & 951 & 0 \\
\hline LA10 & $15 \times 5$ & 958 & 958 & 958 & 0.03 & 0 & 958 & 0.00 & 958 & 0 & 958 & 0 & 958 & 0.00 & 958 & 0 \\
\hline LA11 & $20 \times 5$ & 1222 & 1222 & 1222 & 0.08 & 0 & 1222 & 0.00 & 1222 & 0 & 1222 & 0 & 1222 & 0.00 & 1222 & 0 \\
\hline LA12 & $20 \times 5$ & 1039 & 1039 & 1039 & 0.08 & 0 & 1039 & 0.00 & 1039 & 0 & 1039 & 0 & 1039 & 0.00 & 1039 & 0 \\
\hline LA13 & $20 \times 5$ & 1150 & 1150 & 1150 & 0.08 & 0 & 1150 & 0.00 & 1150 & 0 & 1150 & 0 & 1150 & 0.00 & 1150 & 0 \\
\hline LA14 & $20 \times 5$ & 1292 & 1292 & 1292 & 0.04 & 0 & 1292 & 0.00 & 1292 & 0 & 1292 & 0 & 1292 & 0.00 & 1292 & 0 \\
\hline LA15 & $20 \times 5$ & 1207 & 1207 & 1207 & 0.18 & 0 & 1207 & 0.00 & 1207 & 0 & 1207 & 0 & 1207 & 0.00 & 1207 & 0 \\
\hline LA16 & $10 \times 10$ & 945 & 945 & 945 & 17.67 & 0 & 945 & 0.00 & 945 & 0 & 945 & 0 & 945 & 0.00 & 945 & 0 \\
\hline LA17 & $10 \times 10$ & 784 & 784 & 784 & 1.81 & 0 & 784 & 0.00 & 784 & 0 & 784 & 0 & 784 & 0.00 & 784 & 0 \\
\hline LA18 & $10 \times 10$ & 848 & 848 & 848 & 4.84 & 0 & 848 & 0.00 & 848 & 0 & 848 & 0 & 848 & 0.00 & 848 & 0 \\
\hline LA19 & $10 \times 10$ & 842 & 842 & 842 & 23.48 & 0 & 842 & 0.00 & 842 & 0 & 842 & 0 & 844 & 0.24 & 842 & 0 \\
\hline LA20 & $10 \times 10$ & 902 & 902 & 902 & 33.99 & 0 & 907 & 0.55 & 902 & 0 & 907 & 0.55 & 907 & 0.55 & 902 & 0 \\
\hline LA21 & $15 \times 10$ & 1046 & 1046 & 1046.55 & 150.91 & 0 & 1046 & 0.00 & 1046 & 0 & 1059 & 1.24 & 1046 & 0.00 & 1046 & 0 \\
\hline LA22 & $15 \times 10$ & 927 & 927 & 928.45 & 134.61 & 0 & 927 & 0.00 & 927 & 0 & 932 & 0.54 & 935 & 0.86 & 927 & 0 \\
\hline LA23 & $15 \times 10$ & 1032 & 1032 & 1032 & 0.67 & 0 & 1032 & 0.00 & 1032 & 0 & 1032 & 0 & 1032 & 0.00 & 1032 & 0 \\
\hline LA24 & $15 \times 10$ & 935 & 935 & 938.8 & 208.53 & 0 & 935 & 0.00 & 935 & 0 & 946 & 1.18 & 953 & 1.93 & 935 & 0 \\
\hline LA25 & $15 \times 10$ & 977 & 977 & 977.1 & 211.38 & 0 & 977 & 0.00 & 977 & 0 & 988 & 1.13 & 981 & 0.41 & 977 & 0 \\
\hline LA26 & $20 \times 10$ & 1218 & 1218 & 1218 & 2.83 & 0 & 1218 & 0.00 & 1218 & 0 & 1218 & 0 & 1218 & 0.00 & 1218 & 0 \\
\hline LA27 & $20 \times 10$ & 1235 & 1235 & 1235.85 & 237.79 & 0 & 1235 & 0.00 & 1235 & 0 & 1251 & 1.3 & 1236 & 0.08 & 1235 & 0 \\
\hline LA28 & $20 \times 10$ & 1216 & 1216 & 1216 & 14.02 & 0 & 1216 & 0.00 & 1216 & 0 & 1216 & 0 & 1216 & 0.00 & 1216 & 0 \\
\hline LA29 & $20 \times 10$ & 1152 & 1153 & 1162.25 & 449.38 & 0.09 & 1157 & 0.43 & 1153 & 0.09 & 1179 & 2.34 & 1160 & 0.69 & 1156 & 0.35 \\
\hline LA30 & $20 \times 10$ & 1355 & 1355 & 1355 & 2.02 & 0 & 1355 & 0.00 & 1355 & 0 & 1355 & 0 & 1355 & 0.00 & 1355 & 0 \\
\hline LA31 & $30 \times 10$ & 1784 & 1784 & 1784 & 1.41 & 0 & 1784 & 0.00 & 1784 & 0 & 1784 & 0 & 1784 & 0.00 & 1784 & 0 \\
\hline LA32 & $30 \times 10$ & 1850 & 1850 & 1850 & 1.57 & 0 & 1850 & 0.00 & 1850 & 0 & 1850 & 0 & 1850 & 0.00 & 1850 & 0 \\
\hline LA33 & $30 \times 10$ & 1719 & 1719 & 1719 & 1.42 & 0 & 1719 & 0.00 & 1719 & 0 & 1719 & 0 & 1719 & 0.00 & 1719 & 0 \\
\hline LA34 & $30 \times 10$ & 1721 & 1721 & 1721 & 1.76 & 0 & 1721 & 0.00 & 1721 & 0 & 1721 & 0 & 1721 & 0.00 & 1721 & 0 \\
\hline LA35 & $30 \times 10$ & 1888 & 1888 & 1888 & 2.1 & 0 & 1888 & 0.00 & 1888 & 0 & 1888 & 0 & 1888 & 0.00 & 1888 & 0 \\
\hline LA36 & $15 \times 15$ & 1268 & 1268 & 1268.1 & 190.4 & 0 & - & 0.00 & 1268 & 0 & 1278 & 0.79 & 1287 & 1.50 & 1268 & 0 \\
\hline LA37 & $15 \times 15$ & 1397 & 1397 & 1407 & 220.13 & 0 & - & 0.00 & 1397 & 0 & 1419 & 1.57 & 1407 & 0.72 & 1397 & 0 \\
\hline LA38 & $15 \times 15$ & 1196 & 1196 & 1197.9 & 215.83 & 0 & - & 0.00 & 1196 & 0 & 1204 & 0.67 & 1196 & 0.00 & 1196 & 0 \\
\hline LA39 & $15 \times 15$ & 1233 & 1233 & 1237.3 & 368.5 & 0 & - & 0.00 & 1233 & 0 & 1247 & 1.14 & 1233 & 0.00 & 1233 & 0 \\
\hline LA40 & $15 \times 15$ & 1222 & 1222 & 1224.85 & 309.27 & 0 & - & 0.00 & 1222 & 0 & 1234 & 0.98 & 1229 & 0.57 & 1224 & 0.16 \\
\hline FT06 & $6 \times 6$ & 55 & 55 & 55 & 0.07 & 0 & 55 & 0.00 & 55 & 0 & 55 & 0 & 55 & 0.00 & 55 & 0 \\
\hline FT10 & $10 \times 10$ & 930 & 930 & 930 & 98.44 & 0 & 930 & 0.00 & 930 & 0 & 930 & 0 & 930 & 0.00 & 930 & 0 \\
\hline FT20 & $20 \times 5$ & 1165 & 1165 & 1165 & 25.97 & 0 & 1165 & 0.00 & 1165 & 0 & 1165 & 0 & 1165 & 0.00 & 1165 & 0 \\
\hline$M R E$ & - & - & - & - & - & 0.002 & - & 0.023 & - & 0.002 & - & 0.312 & - & 0.176 & - & 0.012 \\
\hline
\end{tabular}




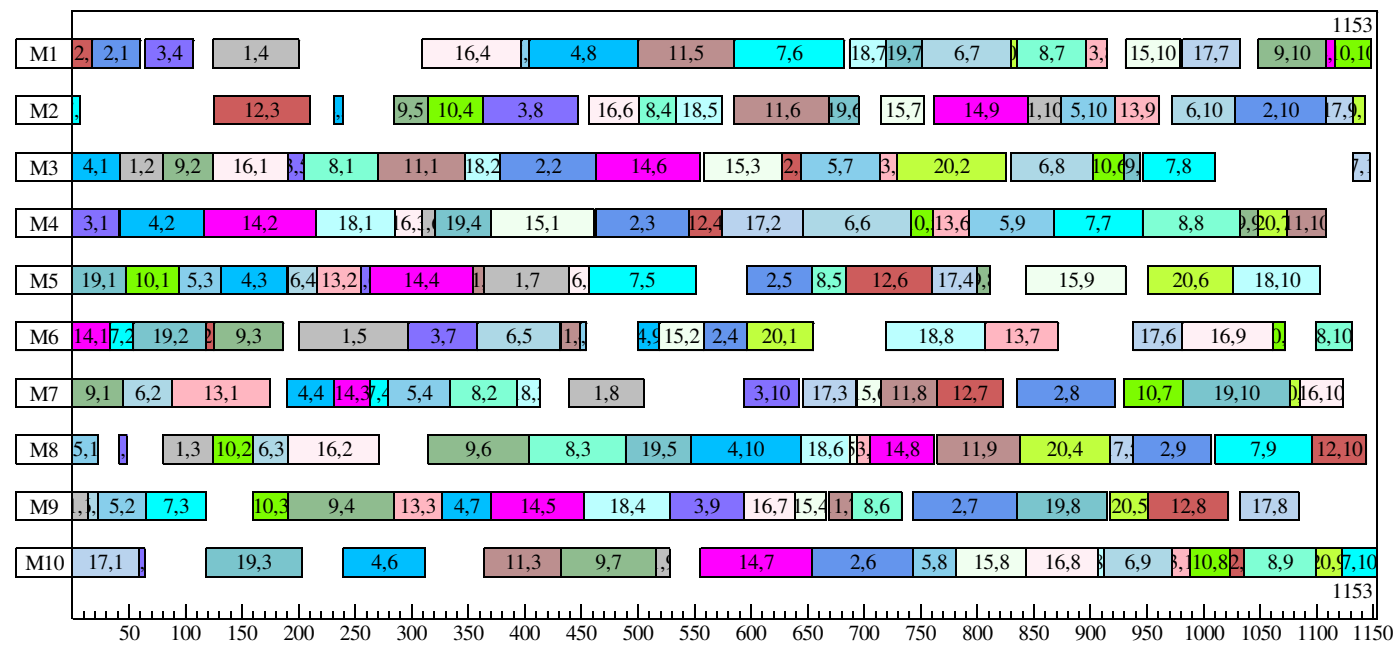

Figure 8 Gantt chart of LA29 instance $\left(C_{\max }=1153\right)$

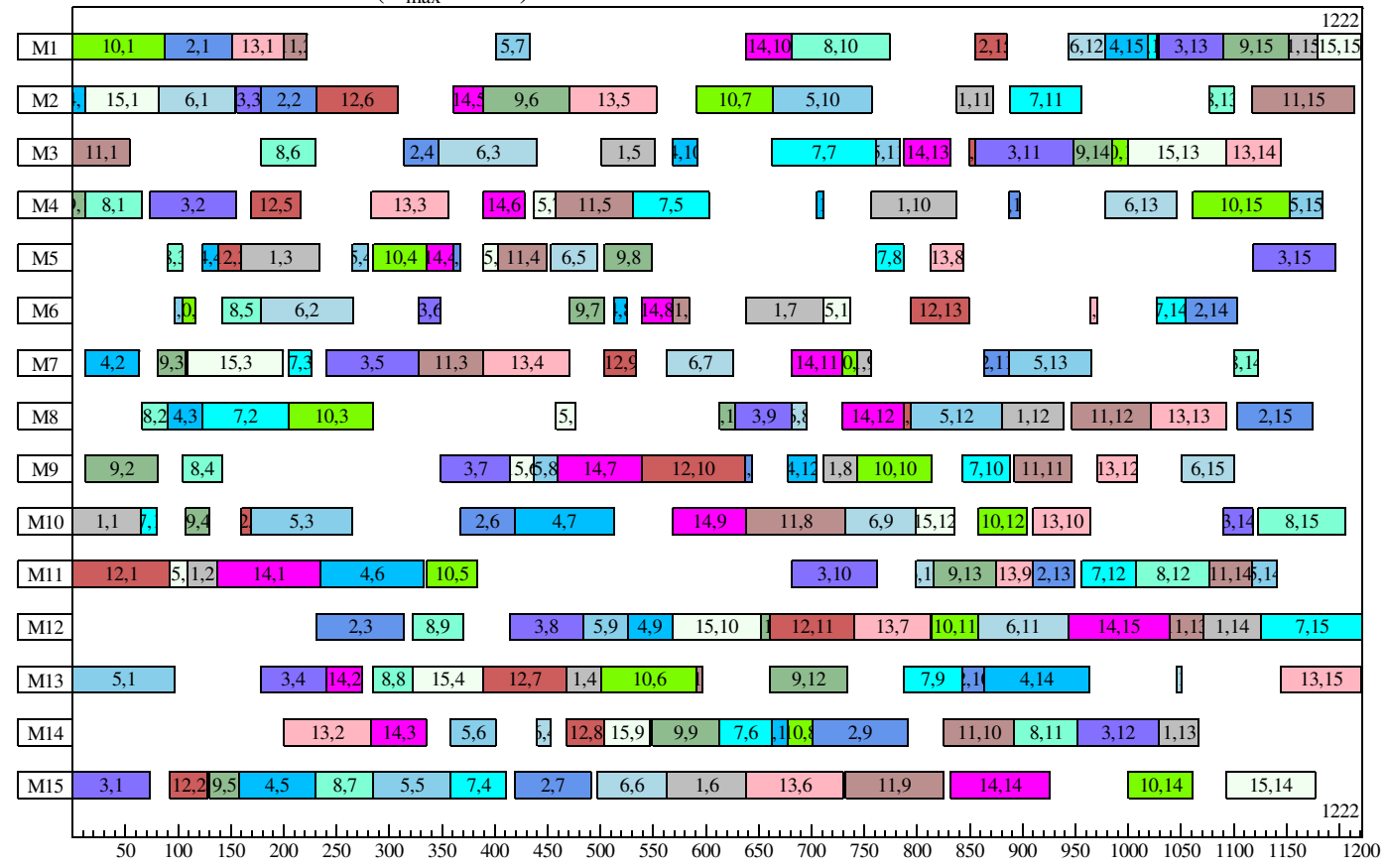

Figure 9 Gantt chart of LA40 instance $\left(C_{\max }=1222\right)$

Table 3 Computational results and comparisons of ORB benchmark instances

\begin{tabular}{|c|c|c|c|c|c|c|c|c|c|c|c|c|c|c|c|c|}
\hline \multirow[t]{2}{*}{ Instance } & \multirow[t]{2}{*}{$n \times m$} & \multirow[t]{2}{*}{$L B$} & \multicolumn{4}{|c|}{ Proposed algorithm } & \multicolumn{2}{|c|}{$\begin{array}{c}\mathrm{TS} / \mathrm{SA} \\
{[5]}\end{array}$} & \multicolumn{2}{|c|}{$\begin{array}{c}\text { BeFABC } \\
{[31]} \\
\end{array}$} & \multicolumn{2}{|c|}{$\begin{array}{r}\mathrm{HPV} \\
{[33]} \\
\end{array}$} & \multicolumn{2}{|c|}{$\begin{array}{c}\mathrm{TS} / \mathrm{PR} \\
{[5]} \\
\end{array}$} & \multicolumn{2}{|c|}{$\begin{array}{c}\text { BRKGA } \\
{[7]}\end{array}$} \\
\hline & & & $C_{\max }$ & $A V\left(C_{\max }\right)$ & $A V(\mathrm{CPU})$ & $R E$ & $C_{\max }$ & $R E$ & $C_{\max }$ & $R E$ & $C_{\max }$ & $R E$ & $C_{\max }$ & $R E$ & $C_{\max }$ & $R E$ \\
\hline ORB01 & $10 \times 10$ & 1059 & 1059 & 1060.8 & 127.03 & 0 & 1059 & 0 & 1059 & 0 & 1059 & 0 & 1059 & 0 & 1059 & 0 \\
\hline ORB02 & $10 \times 10$ & 888 & 888 & 888.35 & 212.27 & 0 & 888 & 0 & 888 & 0 & 888 & 0 & 888 & 0 & 888 & 0 \\
\hline ORB03 & $10 \times 10$ & 1005 & 1005 & 1014.35 & 101.33 & 0 & 1005 & 0 & 1005 & 0 & 1005 & 0 & 1005 & 0 & 1005 & 0 \\
\hline ORB04 & $10 \times 10$ & 1005 & 1005 & 1007.4 & 203.72 & 0 & 1005 & 0 & 1005 & 0 & 1005 & 0 & 1005 & 0 & 1005 & 0 \\
\hline ORB05 & $10 \times 10$ & 887 & 887 & 888.1 & 233.67 & 0 & 887 & 0 & 887 & 0 & 887 & 0 & 887 & 0 & 887 & 0 \\
\hline ORB06 & $10 \times 10$ & 1010 & 1010 & 1010 & 145.68 & 0 & 1010 & 0 & 1010 & 0 & 1010 & 0 & 1010 & 0 & 1010 & 0 \\
\hline ORB07 & $10 \times 10$ & 397 & 397 & 397 & 15.43 & 0 & 397 & 0 & 397 & 0 & 397 & 0 & 397 & 0 & 397 & 0 \\
\hline ORB08 & $10 \times 10$ & 899 & 899 & 901.4 & 157.92 & 0 & 899 & 0 & 899 & 0 & 899 & 0 & 899 & 0 & 899 & 0 \\
\hline ORB09 & $10 \times 10$ & 934 & 934 & 934.25 & 54.26 & 0 & 934 & 0 & 934 & 0 & 934 & 0 & 934 & 0 & 934 & 0 \\
\hline ORB10 & $10 \times 10$ & 944 & 944 & 944 & 4.91 & 0 & 944 & 0 & 944 & 0 & 944 & 0 & 944 & 0 & 944 & 0 \\
\hline$M R E$ & - & - & - & - & - & 0 & - & 0 & - & 0 & - & 0 & - & 0 & - & 0 \\
\hline
\end{tabular}




\section{Conclusions}

(1) Aiming at the job shop scheduling problem, an iterated greedy hybrid algorithm for optimizing the maximum completion time is proposed. The effectiveness of the proposed algorithm is verified by testing international JSP benchmarks.

(2) In the deconstruction and reconstruction of iterated greedy algorithm, the operation-level deconstruction and reconstruction are realized, so that multiple operations can be moved arbitrarily, and a calculation method of the head and tail length of partial scheduling operations based on adjacency matrix and topological sorting is given.

(3) The reconstruction mechanism based on constructing the initial scheduling insertion algorithm is designed, which ensures the feasibility after multiple operations are inserted, and scientifically inserts operations with better strategies, and provides a method reference for studying other similar problems with the characteristics of JSP problem.

(4) The next step is to apply the designed deconstruction and reconstruction mechanism to other extension problems of JSP, expand the scope of application, and integrate with other swarm intelligence optimization algorithms to further improve the algorithm performance.

\section{Declaration}

\section{Acknowledgements}

Not applicable

\section{Funding}

Supported by National Natural Science Foundation of China (Grant No. 51775240)

\section{Availability of data and materials}

The datasets supporting the conclusions of this article are included within the article.

\section{Authors' contributions}

The author' contributions are as follows: Shi-Kui Zhao was in charge of the whole trial; Lin Huang assisted with writing-review and editing; Qing Han assisted with writing-review and grammar check.

\section{Competing interests}

The authors declare no competing financial interests.

\section{Consent for publication \\ Not applicable}

\section{Ethics approval and consent to participate \\ Not applicable}

\section{References}

[1] E D Taillard. Parallel taboo search techniques for the job shop scheduling problem. ORSA Journal on Computing, 1994, 6(2): 108-117.

[2] E Nowicki, C Smutnicki. A fast taboo search algorithm for the job shop problem. Management Science, 1996, 42(6): 797-813.

[3] E Nowicki, C Smutnicki. Some new tools to solve the job-shop problem. Technical Report, Institute of Engineering Cybernetics, Wroclaw University of Technology, Wroclaw, Poland, 2002.

[4] C Y Zhang, P G Li, Z LGuan, et al. A tabu search algorithm with a new neighborhood structure for the job shop scheduling problem. Computers \& Operations Research, 2007, 34(11): 3229-3242.

[5] C Y Zhang, P G Li, Y Q Rao, et al. A very fast TS/SA algorithm for the job shop scheduling problem. Computers \& Operations Research, 2008, 35(1): 282-294.

[6] M M Nasiri, F Kianfar. A GES/TS algorithm for the job shop scheduling. Computers \& Industrial Engineering, 2012, 62(4): 946-952.

[7] J F Gonçalves, M G C Resende. An extended Akers graphical method with a biased random-key genetic algorithm for job-shop scheduling. International Transactions in Operational Research, 2014, 21(2): 215-246.

[8] B Peng, Z P Lü, T C E Cheng. A tabu search/path relinking algorithm to solve the job shop scheduling problem. Computers \& Operations Research, 2015, 53(53): 154-164.

[9] T C E Cheng, B Peng, Z P Lü. A hybrid evolutionary algorithm to solve the job shop scheduling problem. Annals of Operations Research, 2016, 242(2): 223-237.

[10] Y Nagata, I Ono. A Guided Local Search with Iterative Ejections of Bottleneck Operations for the Job Shop Scheduling Problem. Computers \& Operations Research, 2018, 90: 60-71.

[11] S Mahmud, A Abbasi, R K Chakrabortty, et al., Multi-operator communication based differential evolution with sequential Tabu Search approach for job shop scheduling problems. Applied soft computing, 2021,108: 107470.

[12] S K Zhao. Research on path relinking based on non-delay scheduling and backtracking tabu search algorithm of job shop scheduling problem. Journal of Mechanical Engineering, 2021, 57(14), 291-303.

[13] M Mastrolilli, L M Gambardella. Effective neighborhood functions for the flexible job shop problem. Journal of Scheduling, 2000, 3(1): 3-20

[14] M A González, C R Vela, R Varela. Scatter search with path relinking for the flexible job shop scheduling problem. European Journal of Operational Research, 2015, 245(1): 35-45.

[15] J W Ding, Z P Lü, C M Li, et al. A two-individual based evolutionary algorithm for the flexible job shop scheduling problem. The Thirty-Third AAAI Conference on Artificial Intelligence (AAAI-19), 2019: 2262-2271.

[16] X Y Li, L Gao. An effective hybrid genetic algorithm and tabu 
search for flexible job shop scheduling problem. International Journal of Production Economics, 2016, 174, 93-110.

[17] R Ruiz, T Stützle. A simple and effective iterated greedy algorithm for the permutation flowshop scheduling problem. European journal of operational research, 2007, 177(3): 2033-2049.

[18] G Minella, R Ruiz, M Ciavotta. Restarted iterated pareto greedy algorithm for multi-objective flowshop scheduling problems. Computers \& Operations Research, 2011, 38(11): 1521-1533.

[19] Q K Pan, R Ruiz. An effective iterated greedy algorithm for the mixed no-idle permutation flowshop scheduling problem. Omega, 2014, 44: 41-50.

[20] M F Tasgetiren, D Kizilay, Q K Pan, et al. Iterated greedy algorithms for the blocking flowshop scheduling problem with makespan criterion. Computers \& operations research, 2017, 77: 111-126.

[21] W S Shao, D C Pi, Z S Shao. Optimization of makespan for the distributed no-wait flow shop scheduling problem with iterated greedy algorithms. Knowledge-based systems, 2017, 137: 163-181.

[22] R Ruiz, Q K Pan, B Naderi. Iterated greedy methods for the distributed permutation fowshop scheduling problem. Omega, 2019, 83: 213-222.

[23] G L Deng, Q T Su, Z W Zhang, et al. A population-based iterated greedy algorithm for no-wait job shop scheduling with total flow time criterion. Engineering Applications of Artificial Intelligence, 2020, 88: 103369.1-103369.15.

[24] M Pranzo, D Pacciarelli. An iterated greedy metaheuristic for the blocking job shop scheduling problem. Journal of Heuristics, 2016, 22(4): 587-611.

[25] J S Kim, J H Park, D H Lee. Iterated greedy algorithms to minimize the total family flow time for job-shop scheduling with job families and sequence-dependent set-ups. Engineering optimization. 2017, 49(10): 1719-1732.

[26] G A Aqel, X Y Li, L Gao. A modified iterated greedy algorithm for flexible job shop scheduling problem. Chinese Journal of Mechanical Engineering, 2019, 32(2): 157-167.

[27] M Nawaz, E Enscore, J I Ham. A heuristic algorithm for the m-machine, n-job flow-shop sequencing problem. Omega, 1983,
11(1): 91-95.

[28] S K Zhao. A hybrid algorithm with a new neighborhood structure for the job shop scheduling problem. Journal of Mechanical Engineering, 2016, 52(9): 141-150.

[29] E Balas, A Vazacopoulos. Guided local search with shifting bottleneck for job shop scheduling. Management Science, 1998, 44(2): 262-275.

[30] L Asadzadeh. A parallel artificial bee colony algorithm for the job shop scheduling problem with a dynamic migration strategy. Computers \& Industrial Engineering, 2016, 102: 359-367.

[31] N Sharma, H Sharma, A Sharma. Beer froth artificial bee colony algorithm for job-shop scheduling problem. Applied Soft Computing, 2018, 68: 507-524.

[32] R Qing-dao-er-ji, Y P Wang. A new hybrid genetic algorithm for job shop scheduling problem. Computers \& Operations Research, 2012, 39(10): 2291-2299.

[33] L Gao, X Y Li, X Y Wen, et al. A hybrid algorithm based on a new neighborhood structure evaluation method for job shop scheduling problem. Computers \& Industrial Engineering, 2015, 88: 417-429.

\section{Biographical notes}

Shi-Kui Zhao, born in 1984, is currently an associate professor at School of Mechanical Engineering, University of Jinan, China. He received his $\mathrm{PhD}$ degree from Zhejiang Universtiy, China, in 2013. His research interests include shop scheduling and intelligent scheduling optimization.

Tel: +86-531-89736314; E-mail: me_zhaosk@ujn.edu.cn

Lin Huang, born in 1995, is currently a master candidate at School of Mechanical Engineering, University of Jinan, China.

Qing Han, born in 1982, is currently an associate professor at School of Mechanical Engineering, University of Jinan, China. She received her $\mathrm{PhD}$ degree from Zhejiang Universtiy, China, in 2012. 